\title{
Platelet Shape Changes during Thrombus Formation: Role of Actin-Based Protrusions
}

\author{
Markus Bender ${ }^{1}$ Raghavendra Palankar ${ }^{2}$ \\ ${ }^{1}$ Institute of Experimental Biomedicine - Chair I, University Hospital \\ and Rudolf Virchow Center, Würzburg, Germany \\ 2 Department of Transfusion Medicine,Institute for Immunology and \\ Transfusion Medicine, University Medicine Greifswald, Greifswald, \\ Germany
}

\begin{abstract}
Address for correspondence Markus Bender, PhD, Institute of Experimental Biomedicine, Chair I, University Hospital Würzburg, Josef-Schneider-Str. 2, 97080 Würzburg, Germany (e-mail: Bender_M1@ukw.de).
\end{abstract}

Hämostaseologie 2021;41:14-21.

\begin{abstract}
Keywords

- platelet

- thrombus

- cytoskeleton

- lamellipodia

- actin

Platelet activation and aggregation are essential to limit blood loss at sites of vascular injury but may also lead to occlusion of diseased vessels. The platelet cytoskeleton is a critical component for proper hemostatic function. Platelets change their shape after activation and their contractile machinery mediates thrombus stabilization and clot retraction. In vitro studies have shown that platelets, which come into contact with proteins such as fibrinogen, spread and first form filopodia and then lamellipodia, the latter being plate-like protrusions with branched actin filaments. However, the role of platelet lamellipodia in hemostasis and thrombus formation has been unclear until recently. This short review will briefly summarize the recent findings on the contribution of the actin cytoskeleton and lamellipodial structures to platelet function.
\end{abstract}

\section{Introduction}

Platelets are anucleated blood cells and play an invaluable role in hemostasis. After vascular injury, platelets arrest on exposed subendothelial components, become activated, and form a hemostatic plug which is essential to prevent excessive blood loss. However, uncontrolled thrombus formation in diseased vessels may lead to irreversible vessel occlusion and infarction of vital organs. Platelet adhesion and thrombus formation is a multistep process mediated by multiple receptor-ligand interactions. ${ }^{1}$ The initial capture of flowing platelets occurs via the interaction of the platelet glycoprotein (GP) Ib-V-IX complex with immobilized von Willebrand factor on subendothelial collagen. This interaction allows the binding of the receptor GPVI to collagen, thereby initiating platelet activation. ${ }^{2}$ The conformational shift of $\beta 1$ and $\beta 3$ integrins to a high-affinity state finally enables firm platelet adhesion to the subendothelial components and platelet aggregation. As a result, local production of thrombin and the release as well as the synthesis of soluble mediators reinforce and sustain platelet activation. Besides enhancing platelet activation, thrombin also catalyzes the production of activated coagulation factors and converts fibrinogen to fibrin. The fibrin network covers thrombi and determines the thrombus microelasticity. ${ }^{3}$

\section{Platelet Cytoskeleton and Biomechanics}

The cytoskeleton plays a major role in platelet function as it maintains the discoid shape of circulating platelets and dynamically rearranges in response to activation. The platelet cytoskeleton consists of the proteins spectrin, myosin, tubulin, and actin as well as several cytoskeletal-regulatory proteins (-Fig. 1A) ${ }^{4,5}$ Spectrins (approximately 2,000 molecules in a single platelet) form heterodimers ( $\alpha / \beta$ subunits) and assemble into tetramers of $200 \mathrm{~nm}$ lengths. The spectrin-based membrane cytoskeleton interconnects with actin filaments and supports the maintenance of the platelet shape. ${ }^{6,7}$ Microtubules are dynamic, polarized structures and their assembly is regulated by multiple microtubule-associated proteins. Approximately 250,000 tubulin dimers $(\alpha / \beta$ subunits) are present in a single platelet with up to $55 \%$ organized in microtubule filaments. In resting platelets, the microtubules form approximately 8 to 12 characteristic, ring-shaped received

October 13, 2020

accepted after revision

November 28, 2020 (c) 2021. Thieme. All rights reserved. Georg Thieme Verlag KG,

Rüdigerstraße 14,

70469 Stuttgart, Germany
DOI https://doi.org/ 10.1055/a-1325-0993. ISSN 0720-9355. 
A

B
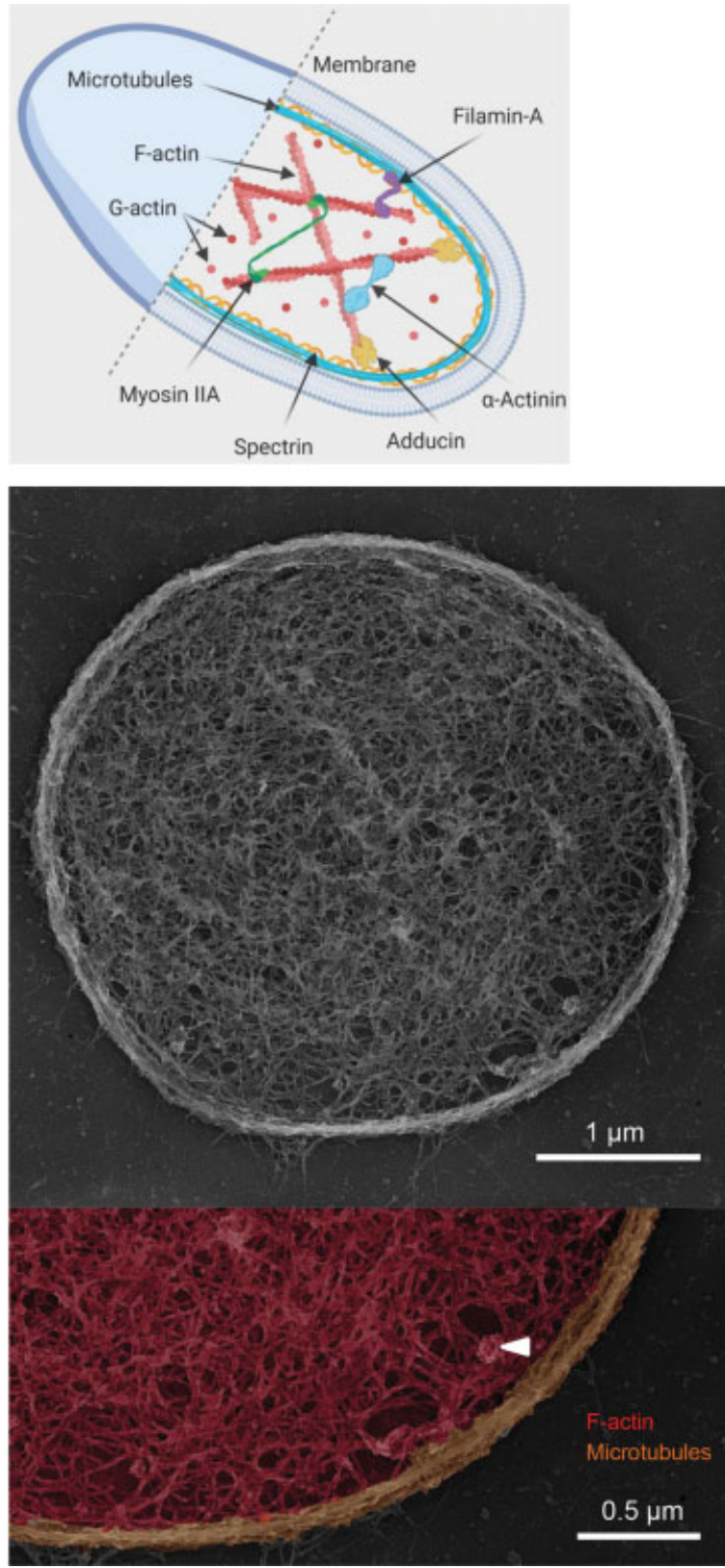

Fig. 1 Platelet cytoskeleton. (A) Scheme of the cytoskeleton of a resting platelet. Created with BioRender.com. (B) Platinum-replica electron microscopy image of the cytoskeleton of a resting mouse platelet on poly-L-lysine. Lower image: pseudo-colored zoom-in image. The arrow head points to a clathrin-coated pit.

microtubule coils (-Fig. 1B), the so-called marginal band, which was described to be maintained by the antagonistic actions of the microtubule-interacting proteins dynein and kinesin. ${ }^{8,9}$ Activated platelets rapidly undergo shape change from discoid to spherical as a result of marginal band coiling due to altered dynein/kinesin activity. ${ }^{10,11}$ Dmitrieff et al examined using in silico simulations how the marginal band elasticity and cortical tension determine platelet morphology, and found that when cortical tension increases faster than cross-linkers can unbind, the marginal band will coil at the onset of platelet activation. ${ }^{12}$ The cross-linked filamentous network of actin cytoskeleton is one of the major structural components of the cell. A single platelet contains approximately 2 million actin monomers (G-actin), which is approximately $15 \%$ of the total protein content. A total of $40 \%$ of the $\mathrm{G}$-actin is polymerized into 2,000 to 5,000 actin filaments (F-actin) building a mechanically rigid cytoplasmic actin network (-Fig. 1B). ${ }^{13,14}$ During platelet activation, the actin cytoskeleton undergoes rapid reorganization regulated by actin-binding proteins and confers multiple functions, including force generation and mechanotransduction. The list of actin-associated and-regulating proteins has grown at a steady pace, thereby increasing our knowledge about the role of the cytoskeleton machinery in platelet function. ${ }^{15}$

Due to their high intracellular actin content and surface receptor density, platelets are considered as ideal cells to mechanosense their environment and transduce cellular signals in response to a myriad of physicochemical and biomechanical stimuli ( - Fig. 2).$^{16}$ Within this context, the platelet actomyosin complex plays a central role during retraction (i.e., volume shrinkage) of newly formed blood clots by compaction of the fibrin network. ${ }^{17}$ Platelets achieve this by mechanically pulling fibrin transversely as well as along their longitudinal axes. ${ }^{18}$ Although the detailed biophysical mechanisms underlying platelet mechanobiology are only poorly explored, recent use of force-sensitive biophysical tools has unraveled the link between platelet mechanotransduction and cytoskeleton-dependent biomechanics at different length scales. For example, with regard to nanomechanics of single-platelet contraction, Myers et al developed a high-throughput hydrogel based platelet-contraction cytometer that uses displacement of fibrinogen microdots and showed that Wiskott-Aldrich syndrome and May-Hegglin Disorder platelets generate reduced contractile forces compared with control platelets. ${ }^{19}$ Similarly, microclot elastometry was used to assess platelet-dependent clot contraction in bulk using shear gradients. It was shown that under these conditions platelet forces are significantly reduced when blood samples are treated among others with inhibitors of myosin, GPIb-IX-V, and integrin $\alpha \operatorname{Ilb} \beta 3 .{ }^{20}$ Implementation of most of these biophysical approaches has been limited to the investigation of adherent platelet biomechanics, since assessment of intrinsic biomechanical properties of nonadherent single platelets in their native environment has remained challenging. To specifically address this, high-throughput real-time deformability cytometry (RT-DC) has been established ${ }^{21}$ and is meanwhile used to study deformability of platelets from genetically modified mice. It was demonstrated that platelets from coactosin-like 1 (Cotl1, an F-actin binding protein) deficient mice (Pf4-Cre/LoxP) are smaller in size but exhibit a softer biomechanical phenotype (i.e., are more deformable) than their wild-type counterparts. Cotl1 mutant platelets also showed markedly reduced adhesion and thrombus formation on von Willebrand factor at high shear, which demonstrated that Cotl1 is essential for GPIb-mediated platelet tethering/adhesion and downstream mechanotransduction. ${ }^{22}$ Furthermore, it was recently shown that RT-DC-based label-free mechanophenotyping can be also used for quality assessment of cellular blood products, including platelets. ${ }^{23}$ For more detailed insights on biophysical methods, which 


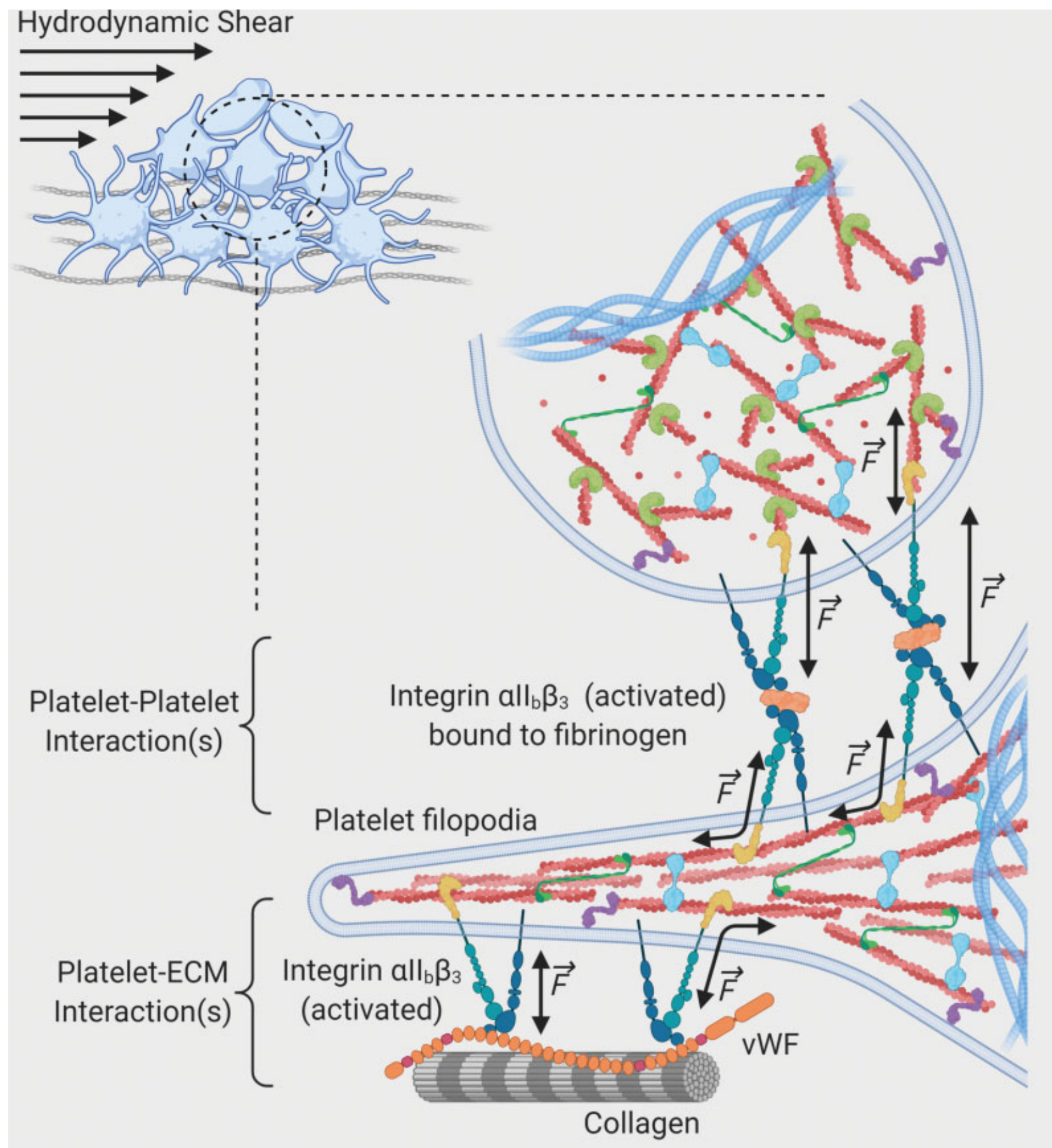

eseosent
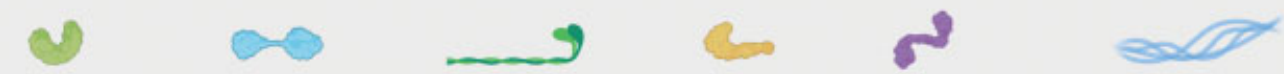

\section{F-actin Arp2/3 a-Actinin Myosin IIA Talin Filamin-A Microtubules}

Fig. 2 Simplified schematic representation of dynamic platelet-extracellular matrix and platelet-platelet interactions governing mechanosensing and thrombus biomechanics. Under hydrodynamic shear after initial platelet tethering, cellular activation and inside-out upregulation of integrin affinity are essential for firm platelet adhesion. Downstream mechanotransductional signaling occurs through coupling of integrins via talin to F-actin generating forces. Activated platelets spread through filopodial extensions with crosslinked F-actin bundles. Released second wave mediators amplify integrin activation on adherent platelets and mediate thrombus growth by activating additional platelets (not depicted). Those platelets are arrested by the activated primary layer of platelets through multiple receptor-ligand interactions (e. g., integrin-fibrinogen). These interactions further amplify signal mechanotransduction at the platelet-platelet interface under hydrodynamic shear and drive thrombus growth and clot compaction. Created with BioRender.com. 
have been applied in platelet biology, the reader is referred to Ciciliano et al, ${ }^{24} \mathrm{Feghhi}$ et al, ${ }^{25}$ and to the recent reviews from Zaninetti et $\mathrm{al}^{26}$ and Sachs et al. ${ }^{27}$

\section{Platelet Spreading under Static Conditions}

Upon platelet activation, a rapid reorganization of the cytoskeleton takes place, which results in platelet shape change and contributes to platelet function. Platelets start to spread under static in vitro conditions when they bind to immobilized adhesive proteins such as fibrinogen. This assay is frequently performed to assess platelet integrin outside-in signaling and the capacity to rearrange the cytoskeletal components. Moreover, static platelet spreading assays have often been used to test the functional properties of in vitro produced platelets. Four distinct stages can be defined during platelet spreading. (1) Platelets start to adhere to the surface, (2) followed by the formation of filopodia, (3) a phase in which filopodia and lamellipodia can be observed, and (4) until only lamellipodia are formed. Filopodia are finger-like protrusions with parallel actin filaments ( - Fig. $3 \mathrm{~A}$ ), whereas lamellipodia are flat, undulating cellular protrusions with orthogonally arrayed short actin filaments ( - Fig. 3B). ${ }^{28}$ Platelets are considered as fully spread when a circumferential zone of orthogonally arrayed short actin filaments within lamellipodia has been formed (-Fig. 3C).

Initial studies in platelets and other cell types have shown that rapid actin polymerization of new filaments at the leading edge of lamellipodia is mediated by $\mathrm{Ca}^{2+}$ gelsolin, which is crucial for actin filament uncapping and severing, and by the small GTPase Rac, which activates the Arp2/3 complex. ${ }^{29}$ The Arp2/3 complex is an important actin filament nucleator and essential to create branched actin filament networks required for formation of lamellipodia. Arp $2 / 3$ is activated by nucleation-promoting factors, the best characterized being Wiskott-Aldrich syndrome protein (WASp) and the WASp-family verprolin-homologous protein (WAVE)-regulatory complex (WRC). ${ }^{30}$ Platelet nodule formation, an F-actin structure present early during spreading and suggested to be important for adhesion processes, was shown to be dependent on the activity of WASp. ${ }^{31}$ However, branching of actin filaments remained functional in fully spread platelets in the absence of WASp. ${ }^{32}$ Interestingly, the WRC subunit cytoplasmic fragile $\mathrm{X}$ mental retardation 1-interacting protein 1 (Cyfip1) has recently been shown to be crucial for formation of branched actin filaments in platelet lamellipodia. ${ }^{33}$ The inactive WRC is activated upon interaction of the subunit Cyfip1 with the small GTPase Rac1. ${ }^{34}$ Subsequently, the active WRC interacts with $\mathrm{Arp} 2 / 3$, and mediates formation of branched actin filaments. Similar to Rac1-deficient ${ }^{35,36}$ and Arp2/3-deficient platelets, ${ }^{37}$ Cyfip1-deficient platelets are unable to reorganize the actin filaments into short, branched actin filaments and therefore do not fully spread. $^{33}$ Taken together, the Rac1-WAVE/Cyfip1-Arp2/3 pathway in platelets plays a crucial role for branching of actin filaments and for lamellipodium formation.

\section{Platelet Shape in Thrombus Formation and Stability}

Blood platelets play a key role in the formation of hemostatic plugs and obstructive thrombi and therefore have to rearrange their cytoskeleton and change their shape. There has been controversial discussion in the literature on the importance of platelet lamellipodial structures in thrombus formation and stability. While lamellipodia can be easily observed under in vitro conditions, it has been challenging to study lamellipodium formation under dynamic in vivo conditions. However, studies on mouse models with deficiencies in the Rac-Cyfip1/WAVE-Arp2/3 complex signaling pathway have contributed to a better understanding on the role of lamellipodia in thrombus formation.

In 2005, McCarty et al investigated the role of the small GTPase Rac in platelet lamellipodium formation. For this, they generated mice with deletion of Rac1, Rac2, and Rac1/Rac2 in the hematopoietic system. ${ }^{35}$ Platelets were allowed to spread on fibrinogen-, collagen-, or laminin-coated surfaces. Rac1- and Rac1/Rac2 double-deficient platelets extended filopodia, but failed to generate lamellipodia. In contrast, Rac2-deficient platelets were comparable to controls. These results demonstrated that Rac1, but not Rac2, is essential for platelet lamellipodium formation. Further, it was revealed that only Rac1 is required for stable thrombus formation under shear flow both ex vivo and in vivo. ${ }^{35}$ In this study, the question was raised whether the increased embolization of Rac1-deficient platelets can be attributed to the defect in lamellipodium formation or to a defect in collagen-induced GPVI signaling. In a later study, Pleines et al followed up on this and demonstrated that Rac1 deficiency in platelets leads to a specific GPVI-dependent phospholipase $\mathrm{C}_{2} 2$ activation defect, resulting in impaired platelet adhesion and defective thrombus formation on collagen under flow, which could be rescued in a co-infusion ex vivo assay using the second wave platelet activators ADP and thromboxane A2. ${ }^{36}$ Based on the results of this study, it was suggested that the insufficient GPVI-mediated platelet activation and release of secondarily acting agonists rather than the inability to form lamellipodia is the major cause for the reduced thrombus stability of $\mathrm{Rac1}^{-/-}$platelets. However, the role of platelet lamellipodia in this process, especially in vivo, was still not sufficiently answered.

To investigate the contribution of the Arp2/3 complex to platelet function, Arpc $2^{f / f l, P F 4-C r e}$ mice (deletion of the p34 subunit encoded by the Arpc2 gene) with a markedly decreased expression of the Arp2/3 complex were analyzed. ${ }^{37}$ These mutant mice displayed a microthrombocytopenia and their platelets generated filopodia but were unable to form lamellipodia on a fibrinogen matrix, demonstrating an essential role of Arp2/3-dependent actin filament branching in lamellipodium formation. Arpc $2^{f / f f, ~ P F 4-C r e ~ m i c e ~ w e r e ~ s u b j e c t e d ~ t o ~ t h e ~ t a i l ~ c l i p ~}$ bleeding assay, which allows the determination of the hemostatic function, and to the $\mathrm{FeCl}_{3}$-induced carotid artery thrombosis assay. Intriguingly, no significant differences were found regarding hemostatic function and thrombus formation. ${ }^{37}$ However, deletion of the Arp2/3 complex subunit 2 (Arpc2) was not completely successful in platelets ( $>95 \%$ reduction), 
A

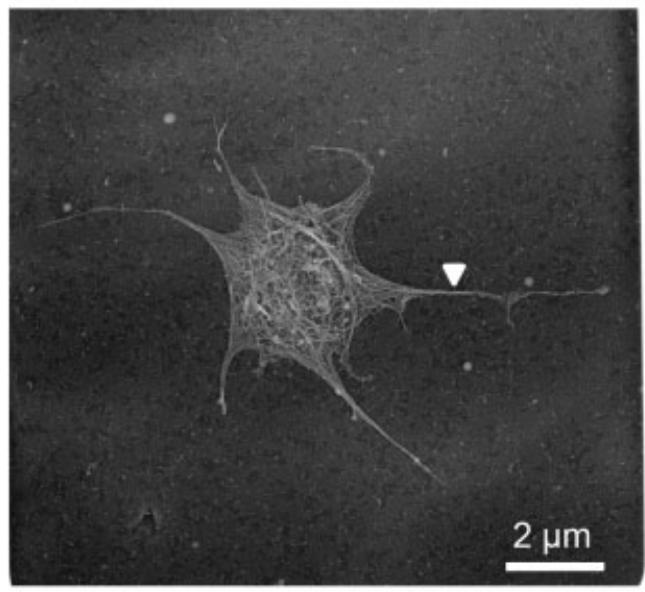

D

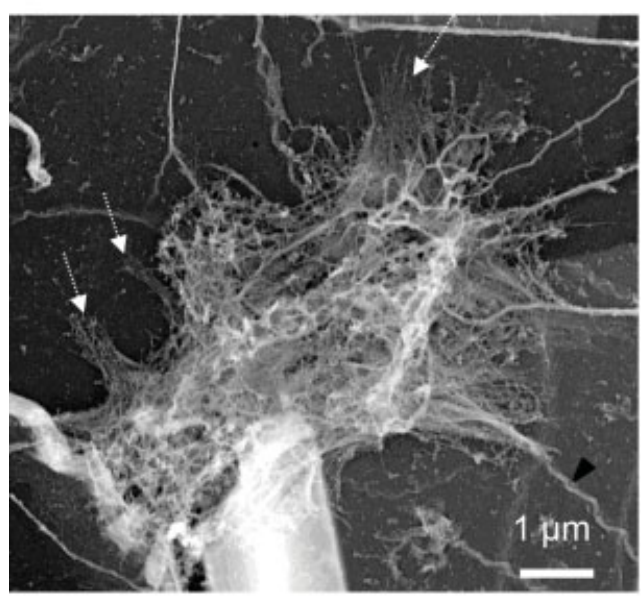

B

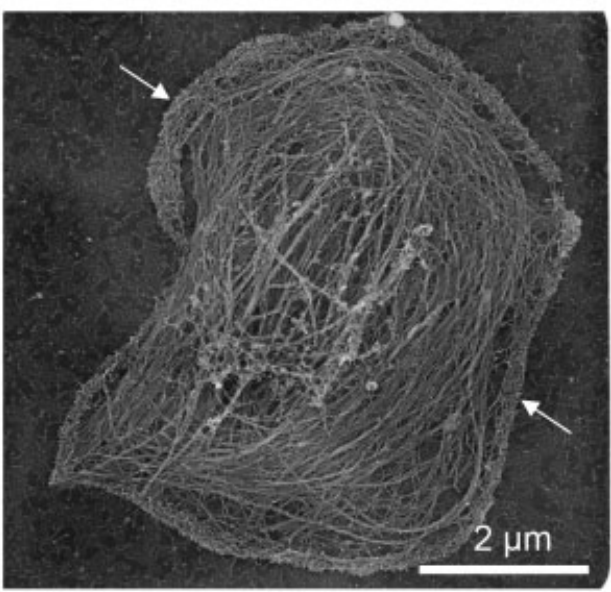

E

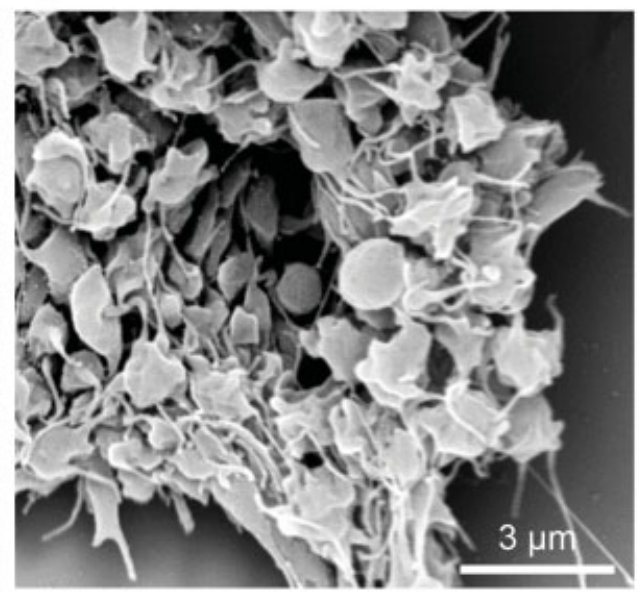

C

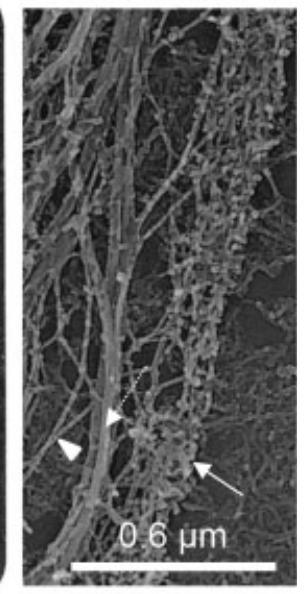

$\mathbf{F}$

Hydrodynamic Shear

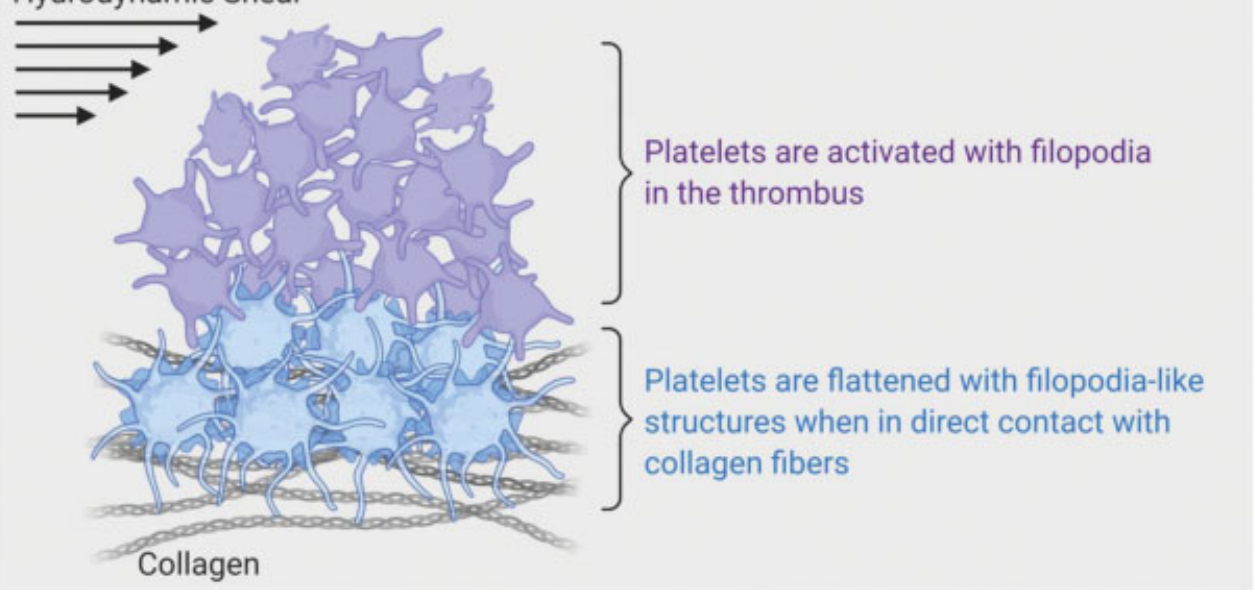

Fig. 3 Platelet shapes under static and dynamic conditions. Cytoskeletal rearrangement of mouse platelets on fibrinogen under static conditions. (A) Platelet filopodia formation. The arrow head points to filopodium with parallel bundles of actin filaments. (B) Platelet lamellipodium formation. The arrows point to the circumferential zone of orthogonally arrayed short filaments. (C) Zoom-in of the peripheral zone of fully spread platelet in (B). White arrow head: long actin filaments; dashed white arrow: microtubules; white arrow: zone of short actin filaments. (D) Platelet with filopodium-like structures (dashed white arrows) when in direct contact to collagen fibers (black arrow head) at the bottom of a thrombus. (E) Scanning electron microscopy image of platelets in a thrombus. (F) Schematic illustration of the platelet shape in a collagen-induced thrombus. Created with BioRender.com. 
which could potentially rescue platelet function. Thus, the role of lamellipodia in this process was still unclear and direct proof was lacking.

Recently, we analyzed platelet-specific Cyfip1 knockout mice to investigate the role of Cyfip1 in platelet function. ${ }^{33}$ This mutant mouse line displayed a normal platelet count and a slight reduction in platelet size. However, lamellipodium formation of mutant platelets was abrogated on all adhesive matrices, such as fibrinogen, collagen IV, collagenrelated peptide, and laminin. Activation of Cyfip1-deficient platelets was only moderately reduced but a selective GPVIsignaling defect could be excluded. ${ }^{33}$ In contrast, the mouse models of Rac 1 and Arp2/3 deficiency described above displayed severe defects in GPVI signaling ${ }^{36}$ and in platelet production as well as incomplete knockout efficiency, ${ }^{35}$ respectively. Thus, without having those side effects, the Cyfip1 knockout mouse line represents a unique mouse model to study the role of lamellipodia in the hemostatic function and thrombus formation. ${ }^{33}$ Bleeding times after tail-tip amputation and thrombus growth in different experimentally induced arterial thrombosis models were comparable between control and Cyfip1-deficient mice showing that lamellipodial structures are not required for classical hemostatic function and pathological thrombus formation. Interestingly, investigation of wild-type platelet morphology revealed that in general platelets with circumferential lamellipodia, equivalent to fully spread platelets in the static spreading assay, could not be observed under flow ex vivo. Platelets under those dynamic conditions were flattened and contained filopodial structures and parallel actin bundles when bound to collagen fibers at the bottom of the thrombus ( Fig. 3D). ${ }^{33}$ Moreover, it was observed that platelets in the thrombus shell ex vivo only formed filopodia, whereas platelike protrusions were absent (-Fig. 3E). Similarly, analysis of the platelet shape in the thrombus shell after experimentally induced in vivo thrombus formation revealed that platelets do not form lamellipodia. Thus, it can be concluded that platelets use only filopodial structures to adhere to collagen fibers and pull on fibrin for clot retraction. Taken together, these results demonstrate that (1) changes in platelet morphology are profoundly different between static and dynamic conditions and (2) that lamellipodium formation is not required for formation and stabilization of a physiological hemostatic plug or pathological thrombus (-Fig. 3F).

\section{Discussion}

Blood platelets are structurally simple cells with a high load of actin, which can be quickly rearranged upon stimulation. Activated platelets are able to generate contractile forces and pull at fibrin fibers resulting in reduction of the clot volume. ${ }^{24}$ To achieve this, platelets require functional surface receptors, signaling pathways, and contractile proteins. However, while the role of platelets in preventing bleeding is well characterized from a biological perspective, the mechanobiological aspects are still only poorly understood. Therefore, additional studies are needed to better understand and quantitatively describe the biomechanical aspects of platelets not only in hemostasis and thrombosis, but also in other platelet-mediated processes. It is envisioned that a comprehensive analysis of platelet cytoskeleton-dependent biomechanical properties using cutting-edge biophysical tools will have diagnostic and prognostic applications: potentially, biophysical characteristics of platelets may be used for the prediction of bleeding risk, risk stratification depending on type of cytoskeletal defect, and thus may also be helpful in determination of clinical need for prophylactic or therapeutic drug intervention and platelet transfusions. $^{26}$

Platelets can extend two different types of cellular protrusions, namely filopodia and lamellipodia, in a static spreading assay. It has been generally believed that platelet filopodial structures are important for sensing the extracellular environment, whereas lamellipodial structures are rather important for sealing the wound and propagating thrombus formation. However, controversial data have been published on the role of lamellipodia in the context of hemostasis and thrombosis. ${ }^{35-37}$ Recent data provide compelling evidence that platelets, which are unable to fully spread and adopt the "fried egg" shape, can perfectly form hemostatic plugs and occlusive thrombi ex vivo and in vivo. ${ }^{33}$ Most likely, the short time window between single platelet adhesion and initiation of thrombus formation does not allow extensive cytoskeletal rearrangement to generate lamellipodia.

However, it has been reported that other platelet shapes play a role in hemostasis. It was shown that collagen-adherent platelets can transform into phosphatidylserine-exposing balloon-like structures, and thereby contribute to thrombin generation and localized coagulation. ${ }^{38}$ Cyfip1-deficient platelets were able to regulate coagulant activity. ${ }^{33}$ This shows that the process of platelet membrane ballooning does not require normal lamellipodium formation.

But what is then the role of lamellipodia in platelet function? It is well known that these structures are important for migration of other cell types. ${ }^{39}$ Gaertner et al showed that platelets migrate to sites of infection to help trap bacteria and clear the vascular surface. ${ }^{40}$ Inhibition of actin polymerization and branching stopped platelet migration in vitro indicating that migration depends on branched lamellipodial actin networks. ${ }^{40}$ The same group also shows that haptotaxis plays a critical role in vascular surveillance during inflammation and infection in vivo. Lamellipodium-dependent migrating platelets scan the vascular endothelium for inflammatory microlesions and plug them, thereby preventing inflammatory microbleeds and suppressing bacterial dissemination. ${ }^{41}$ Interestingly, Gupta et al $^{42}$ observed that platelets are also important to maintain the vascular homeostasis without an injury or inflammation. Absence of platelets, GPVI, PLC 2 , or dense granules increased extravasation of $40-\mathrm{kDa}$ dextran from capillaries and postcapillary venules. Although the authors currently do not favor the hypothesis that platelets directly block local mini-leaks, it is tempting to speculate that platelet shape change might contribute to this process.

In general, further studies will be required to understand more about lamellipodial structures in platelet-related processes. It is also important to identify novel molecular regulators of lamellipodium formation. Is it possible to support migration of 
platelets to sites of inflamed endothelium? We are just about to start understanding the contribution of platelet structures and forces in platelet function.

\section{Conflict of Interest}

The authors declare that they have no conflict of interest.

\section{Acknowledgments}

This work was supported by the Deutsche Forschungsgemeinschaft project number $374031971-$ CRC/TR 240 project A06 to M.B. and R.P., and BE5084/3-2 (DFG-Emmy Noether grant) to M.B. We would like to acknowledge Daniela Naumann and Dr. Yvonne Schurr who performed scanning electron microscopy of platelets, and Dr. Timo Vögtle for proofreading the manuscript. Schematic images were created with BioRender.com.

\section{References}

1 Nieswandt B, Pleines I, Bender M. Platelet adhesion and activation mechanisms in arterial thrombosis and ischaemic stroke. J Thromb Haemost 2011;9(Suppl 1):92-104

2 Vögtle T, Cherpokova D, Bender M, Nieswandt B. Targeting platelet receptors in thrombotic and thrombo-inflammatory disorders. Hamostaseologie 2015;35(03):235-243

3 Swieringa F, Baaten CC, Verdoold R, et al. Platelet control of fibrin distribution and microelasticity in thrombus formation under flow. Arterioscler Thromb Vasc Biol 2016;36(04):692-699

4 Falet H. Anatomy of the platelet cytoskeleton. In: Gresele P, Kleiman NS, Lopez JA, Page CP, eds. Platelets in Thrombotic and Non-Thrombotic Disorders: Pathophysiology, Pharmacology and Therapeutics: an Update. New York, NY: Springer International Publishing AG; 2017:139-156

5 Thomas SG. The structure of resting and activated platelets. In: Michelson AD, ed. Platelets. 4th ed. Cambridge, MA: Elsevier; 2019:47-77

6 Hartwig JH, DeSisto M. The cytoskeleton of the resting human blood platelet: structure of the membrane skeleton and its attachment to actin filaments. J Cell Biol 1991;112(03):407-425

7 Patel-Hett S, Wang H, Begonja AJ, et al. The spectrin-based membrane skeleton stabilizes mouse megakaryocyte membrane systems and is essential for proplatelet and platelet formation. Blood 2011;118(06):1641-1652

8 Italiano JE Jr, Bergmeier W, Tiwari S, et al. Mechanisms and implications of platelet discoid shape. Blood 2003;101(12):4789-4796

9 Steiner M, Ikeda Y. Quantitative assessment of polymerized and depolymerized platelet microtubules. Changes caused by aggregating agents. J Clin Invest 1979;63(03):443-448

10 Diagouraga B, Grichine A, Fertin A, Wang J, Khochbin S, Sadoul K. Motor-driven marginal band coiling promotes cell shape change during platelet activation. J Cell Biol 2014;204(02):177-185

11 Sadoul K. New explanations for old observations: marginal band coiling during platelet activation. J Thromb Haemost 2015;13 (03):333-346

12 Dmitrieff S, Alsina A, Mathur A, Nédélec FJ. Balance of microtubule stiffness and cortical tension determines the size of blood cells with marginal band across species. Proc Natl Acad Sci U S A 2017; 114(17):4418-4423

13 Hartwig J, Italiano J Jr. The birth of the platelet. J Thromb Haemost 2003;1(07):1580-1586

14 Hartwig JH. The platelet: form and function. Semin Hematol 2006; 43(01, Suppl 1):S94-S100

15 Cerecedo D. Platelet cytoskeleton and its hemostatic role. Blood Coagul Fibrinolysis 2013;24(08):798-808
16 Qiu Y, Ciciliano J, Myers DR, Tran R, Lam WA. Platelets and physics: how platelets "feel" and respond to their mechanical microenvironment. Blood Rev 2015;29(06):377-386

17 Weisel JW. Biophysics. Enigmas of blood clot elasticity. Science 2008;320(5875):456-457

18 Kim OV, Litvinov RI, Alber MS, Weisel JW. Quantitative structural mechanobiology of platelet-driven blood clot contraction. Nat Commun 2017;8(01):1274

19 Myers DR, Qiu Y, Fay ME, et al. Single-platelet nanomechanics measured by high-throughput cytometry. Nat Mater 2017;16 (02):230-235

20 Ting LH, Feghhi S, Taparia N, et al. Contractile forces in platelet aggregates under microfluidic shear gradients reflect platelet inhibition and bleeding risk. Nat Commun 2019;10(01):1204

21 Otto O, Rosendahl P, Mietke A, et al. Real-time deformability cytometry: on-the-fly cell mechanical phenotyping. Nat Methods 2015;12(03):199-202, 4, 202

22 Scheller I, Stritt S, Beck S, et al. Coactosin-like 1 integrates signaling critical for shear-dependent thrombus formation in mouse platelets. Haematologica 2020;105(06):1667-1676

23 Aurich K, Fregin B, Palankar R, et al. Label-free on chip quality assessment of cellular blood products using real-time deformability cytometry. Lab Chip 2020;20(13):2306-2316

24 Ciciliano JC, Tran R, Sakurai Y, Lam WA. The platelet and the biophysical microenvironment: lessons from cellular mechanics. Thromb Res 2014;133(04):532-537

25 Feghhi S, Sniadecki NJ. Mechanobiology of platelets: techniques to study the role of fluid flow and platelet retraction forces at the micro- and nano-scale. Int J Mol Sci 2011;12(12):9009-9030

26 Zaninetti C, Sachs L, Palankar R. Role of platelet cytoskeleton in platelet biomechanics: current and emerging methodologies and their potential relevance for the investigation of inherited platelet disorders. Hamostaseologie 2020;40(03):337-347

27 Sachs L, Denker C, Greinacher A, Palankar R. Quantifying singleplatelet biomechanics: an outsider's guide to biophysical methods and recent advances. Res Pract Thromb Haemost 2020;4(03): 386-401

28 Hartwig JH. Mechanisms of actin rearrangements mediating platelet activation. J Cell Biol 1992;118(06):1421-1442

29 Falet H, Hoffmeister KM, Neujahr R, et al. Importance of free actin filament barbed ends for Arp2/3 complex function in platelets and fibroblasts. Proc Natl Acad Sci U S A 2002;99 (26):16782-16787

30 Campellone KG, Welch MD. A nucleator arms race: cellular control of actin assembly. Nat Rev Mol Cell Biol 2010;11(04):237-251

31 Poulter NS, Pollitt AY, Davies A, et al. Platelet actin nodules are podosome-like structures dependent on Wiskott-Aldrich syndrome protein and ARP2/3 complex. Nat Commun 2015; 6:7254

32 Falet H, Hoffmeister KM, Neujahr R, Hartwig JH. Normal Arp2/3 complex activation in platelets lacking WASp. Blood 2002;100 (06):2113-2122

33 Schurr Y, Sperr A, Volz J, et al. Platelet lamellipodium formation is not required for thrombus formation and stability. Blood 2019; 134(25):2318-2329

34 Derivery E, Lombard B, Loew D, Gautreau A. The Wave complex is intrinsically inactive. Cell Motil Cytoskeleton 2009;66(10): 777-790

35 McCarty OJ, Larson MK, Auger JM, et al. Rac1 is essential for platelet lamellipodia formation and aggregate stability under flow. J Biol Chem 2005;280(47):39474-39484

36 Pleines I, Elvers M, Strehl A, et al. Rac1 is essential for phospholipase C-gamma2 activation in platelets. Pflugers Arch 2009;457 (05):1173-1185

37 Paul DS, Casari C, Wu C, et al. Deletion of the Arp2/3 complex in megakaryocytes leads to microthrombocytopenia in mice. Blood Adv 2017;1(18):1398-1408 
38 Agbani EO, van den Bosch MT, Brown E, et al. Coordinated membrane ballooning and procoagulant spreading in human platelets. Circulation 2015;132(15):1414-1424

39 Krause M, Gautreau A. Steering cell migration: lamellipodium dynamics and the regulation of directional persistence. Nat Rev Mol Cell Biol 2014;15(09):577-590

40 Gaertner F, Ahmad Z, Rosenberger G, et al. Migrating platelets are mechano-scavengers that collect and bundle bacteria. Cell 2017; 171(06):1368.e23-1382.e23
41 Nicolai L, Schiefelbein K, Lipsky S, et al. Vascular surveillance by haptotactic blood platelets in inflammation and infection. Nat Commun 2020;11(01):5778

42 Gupta S, Konradt C, Corken A, et al. Hemostasis vs. homeostasis: platelets are essential for preserving vascular barrier function in the absence of injury or inflammation. Proc Natl Acad Sci U S A 2020;117(39):24316-24325 